\title{
Periodic Points for Sphere Maps Preserving Monopole Foliations
}

\author{
Grzegorz Graff $^{1}$ (D) Michał Misiurewicz ${ }^{2} \cdot$ Piotr Nowak-Przygodzki $^{3}$
}

Received: 21 March 2018 / Accepted: 12 November 2018 / Published online: 27 November 2018

(c) The Author(s) 2018

\begin{abstract}
Let $\mathbb{S}^{2}$ be a two-dimensional sphere. We consider two types of its foliations with one singularity and maps $f: \mathbb{S}^{2} \rightarrow \mathbb{S}^{2}$ preserving these foliations, more and less regular. We prove that in both cases $f$ has at least $|\operatorname{deg}(f)|$ fixed points, where $\operatorname{deg}(f)$ is a topological degree of $f$. In particular, the lower growth rate of the number of fixed points of the iterations of $f$ is at least $\log |\operatorname{deg}(f)|$. This confirms the Shub's conjecture in these classes of maps.
\end{abstract}

Keywords Sphere maps $\cdot$ Periodic points $\cdot$ Topological degree $\cdot$ Smooth maps

Mathematics Subject Classification Primary 37E30 $\cdot 37$ C25

\section{Introduction}

Estimating the growth rate of the number of fixed points of the $n$th iterate of a smooth map of the $n$-dimensional sphere to itself is a challenging problem. It was conjectured by Michael Shub in 1974 that it must be (asymptotically) exponential (cf. [17,18]):

\footnotetext{
We would like to thank the anonymous referee for his/her valuable suggestions which helped us to improve the paper. Research supported by the National Science Centre, Poland, UMO-2014/15/B/ST1/01710. Research of Michał Misiurewicz partially supported by Grant No. 426602 from the Simons Foundation.
}

$凶$ Grzegorz Graff

grzegorz.graff@pg.edu.pl

Michał Misiurewicz

mmisiure@math.iupui.edu

Piotr Nowak-Przygodzki

piotrnp@wp.pl

1 Faculty of Applied Physics and Mathematics, Gdańsk University of Technology, Narutowicza 11/12, 80-233 Gdańsk, Poland

2 Department of Mathematical Sciences, Indiana University-Purdue University Indianapolis, 402 N. Blackford Street, Indianapolis, IN 46202, USA

3 Sopot, Poland

Birkhäuser 


$$
\limsup _{n \rightarrow \infty} \frac{\log \# \operatorname{Fix}\left(f^{n}\right)}{n} \geq \log |\operatorname{deg}(f)|
$$

where $\operatorname{deg}(f)$ denotes the degree of $f$.

On the other hand, (1.1) does not hold for every continues map $f$ (cf. an example of a map with only two periodic points and $\operatorname{deg}(f)=2$ in [19]), while it is known that the smoothness implies that the growth of number of periodic orbits with the periods not greater than $n$ is at least linear with respect to $n$ [1] and indeed could be linear up to any fixed period [5] (in particular, cf. [6] for self-maps of $\mathbb{S}^{3}$ ). Proving or disproving the Shub's conjecture would substantially extend our knowledge about the role of the differentiability assumption in periodic point theory.

Even in the simplest case of two-dimensional sphere $\mathbb{S}^{2}$ the conjecture still remains unsolved, although there has been a lot of progress in some particular cases. During the last few years the exponential growth was obtained under some topological conditions for $\mathbb{S}^{2}$ and annulus [2,9-12]. Let us remark that the Shub's question is also interesting if posed in $C^{0}$ topology under some additional conditions on the considered class of maps.

Recently the problem for $\mathbb{S}^{2}$ has been studied in $[15,16]$ (see also $[7,8]$ for higher dimensional spheres) under the assumption that the map is smooth and preserves some "geographical" (singular) foliation. In this context a natural question arises, what happens when we change the "geography" and replace the assumption of smoothness by another one.

In this paper we consider foliations with one singularity and give the description of maps preserving such foliations, revealing mechanisms responsible for appearance of periodic points. In geographical terms, one singularity means that our planet, call it Monopole, has only one pole. After all, if physicists can admit magnetic monopoles (see, e.g. [4]), there is no reason for not admitting geographical monopoles.

The natural coordinates on the surface of Monopole are easy to describe. Suppose that our sphere $\mathbb{S}^{2}$ is given by the equation $x^{2}+y^{2}+(z-1)^{2}=1$. The pole $P$ is the origin. Let us denote the $x y$-plane by $\pi$. A given point $Q=(x, y, z) \in \mathbb{S}^{2}$ belongs to the half plane $\pi_{x}$ containing the $x$-axis and to the half-plane $\pi_{y}$ containing the $y$-axis. We denote by $\alpha(\beta)$ the angle between $\pi_{x}$ and $\pi$ ( $\pi_{y}$ and $\pi$, respectively). We introduce $\alpha$ and $\beta$ as new coordinates of $Q$, both varying from $0^{\circ}$ to $180^{\circ}$. We will call them $y$-titude and $x$-titude.

Thus, the lines of constant $x$-titude (respectively, $y$-titude), which we call merallels (respectively, paridians), are circles that are the intersections of the sphere with the planes passing through the $y$-axis (respectively, the $x$-axis).

Locally, in a neighborhood of the pole, we can easily draw the foliation by the paridians. For this, we use the stereographic projection from the antipole $(0,0,2)$. The picture will be on the $x y$-plane, and the projections of the paridians will be the $x$-axis and the circles tangent to it at the origin (see Fig. 1). We will consider paridianal maps of the sphere, that is, maps preserving the foliation by the paridians.

We also propose a much less regular foliation, with two rabbit-like "ears." The corresponding picture in the $x y$-plane will be the same as for the $y$-paridianal case in 
Fig. 1 Paridianal foliation

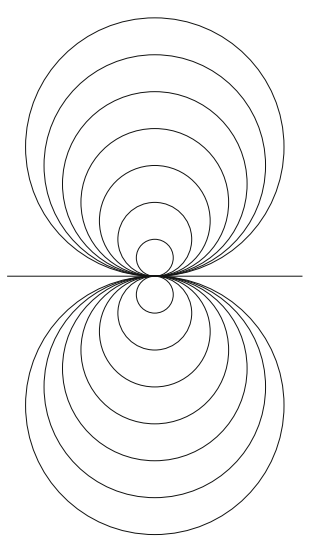

Fig. 2 Rabbit foliation

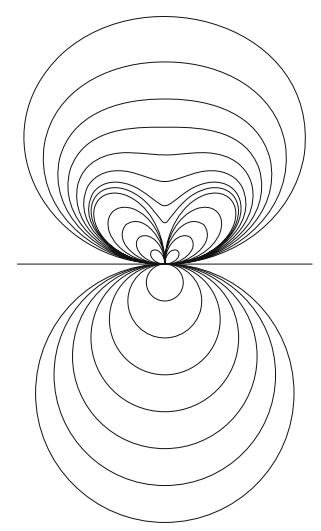

the lower half-plane. In the upper half-plane the leaves will be graphs given by the polar equation $r=c|\sin (2 \theta)|$, where $\theta \in(0, \pi]$, and then the smooth deformations of the circles, being more and more geometrically circle-like as the radius goes to the infinity (see Fig. 2). We will call this foliation the rabbit foliation, and the maps preserving this foliation the rabbit maps.

In our approach we consider some type of open subsets of the base of our foliation with non-zero Brouwer degree called preband and prove a "fixed point theorem" stating the existence of a fixed point of $f$ (or $f^{n}$ for iterations) in every preband. Now in case of homogeneous foliation (paridianal maps), under the assumption that the pole is a sink (which is satisfied if the map is smooth), we get that exponential growth of global degree of iterations implies the same growth of the number of prebands, thus also the number of fixed points of $f^{n}$, so the Shub's conjecture is valid (Theorem 2.19). In fact, we obtain a more precise estimation \# $\operatorname{Fix}\left(f^{n}\right) \geq|\operatorname{deg}(f)|^{n}+1$ if $|\operatorname{deg}(f)|>1$.

If the foliation is not homogeneous, i.e., we consider rabbit maps, the answer is the same. The Shub's conjecture holds, but for a completely different reason: all maps preserving such foliation have degree 0 or \pm 1 (Theorem 3.5). 


\section{Paridianal Maps}

We will use the general scheme from [15]. However, many elements of this scheme, in particular the discussion of the behavior of our map close to the pole, will be different. We use the classical definition of Brouwer degree, cf. [14], where the degree for a $C^{1}$ map $f$ is defined as a sum of signs of the Jacobian of $f$ at a finite set $f^{-1}(y)$ for $y$ being a regular value.

We consider the natural system of coordinates on the sphere $\mathbb{S}^{2}$, defined in the introduction. We include the pole $P$, so the $y$-titude (after the affine rescaling) is an element of the circle $\mathbb{T}=\mathbb{R} / \mathbb{Z}$. To denote specific points on this circle we will usually use the numbers from $[0,1)$. We will denote the $y$-titude of a point $x \in \mathbb{S}^{2}$ by $\ell(x)$. The function $\ell$ is well defined and continuous on $\mathbb{S}^{2} \backslash\{P\}$.

Observe that all paridians, except for the pole, are circles, so the pole belongs to all paridians. However, the singleton of $P$ is also a paridian. We will call paridians other than $\{P\}$ proper paridians. Note that if we used the convention that the pole belongs only to the paridian $\{P\}$, our class of maps preserving paridians would be much smaller.

Definition 2.1 A map $f: \mathbb{S}^{2} \rightarrow \mathbb{S}^{2}$ will be called paridianal if the image of each paridian is contained in a paridian.

Lemma 2.2 Let $f$ be a paridianal map. If $\operatorname{deg}(f) \neq 0$ then $f(P)=P$.

Proof If $f(P) \neq P$ then by the continuity of $f$, since every paridian contains $P$, the image of the whole sphere would be contained in one paridian. Therefore $f$ would not be surjective and thus $\operatorname{deg}(f)=0$.

From now on, we will assume that $\operatorname{deg}(f) \neq 0$.

Fix a paridianal map $f$. Since $f$ maps paridians to paridians, there exists a map $\varphi: \mathbb{T} \rightarrow \mathbb{T}$ such that for $x, f(x) \neq P$

$$
\varphi \circ \ell=\ell \circ f
$$

As we will consider only maps with non-zero degree, by Lemma 2.2 we may assume that $f(P)=P$. Since $P$ belongs to all paridians, $\varphi$ is not defined uniquely. To make it unique, whenever the whole paridian $\ell^{-1}(y)$ is mapped by $f$ to $P$, we set $\varphi(y)=0$ and define $\ell(P)=0$. Then we get the following commutative diagram:

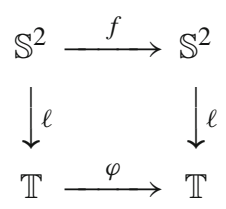

While we cannot claim that $\varphi$ is continuous everywhere, it is continuous where it is important from the point of view of our considerations (cf. Lemma 2.4 below).

Denote $A=\varphi^{-1}(0)$. Let us take $y \notin A$. Then $S_{y}:=\ell^{-1}(y)$ is a circle. Observe that we have orientation of the proper paridians consistent throughout the sphere. The 
map $f$ restricted to the circle $S_{y}$ leads from the circle to the circle. Let us denote the degree of this map by $d_{S_{y}}(f)$. Now consider $B$, a connected component of the set $\mathbb{T} \backslash A$.

Lemma 2.3 For $y \in B$, the degree $d_{S_{y}}(f)$ depends only on $B$, not on $y$.

Proof Let us take a parametrization $\eta_{y}: \mathbb{S}^{1} \rightarrow S_{y}$, which depends smoothly on $y$. Then, $d_{S_{y}}(f)$ is the degree of the self-map of the circle

$$
f_{y}=\left.\eta_{\varphi(y)}^{-1} \circ f\right|_{S_{y}} \circ \eta_{y}
$$

As a consequence by changing $y$ we obtain a homotopy between $f_{y^{\prime}}$ and $f_{y^{\prime \prime}}$ for all $y^{\prime}, y^{\prime \prime} \in B$ and the result follows from homotopical invariance of the degree.

We will call the common value of $d_{S_{y}}(f)$ for $y \in B$ the paridianal degree of $B$, and denote it $d(B)$.

Lemma 2.4 If $d(B) \neq 0$ then $\varphi$ is continuous on the closure of $B$.

Proof Assume that $d(B) \neq 0$ and $B=\left(y^{-}, y^{+}\right)$. Continuity of $\varphi$ on $B$ follows from continuity of $f$. We will show that for $y>y^{-}$which tends to $y^{-}, \varphi(y)$ tends to 0 , i.e., we have right-continuity at $y^{-}$(the argument for left-continuity at $y^{+}$is the same). Notice that $f\left(S_{y}\right)=S_{\varphi(y)}$ and that $f\left(S_{y}\right)$ covers the whole $S_{\varphi(y)}$ because $d(B) \neq 0$. By continuity of $f, f\left(S_{y}\right)\left(=S_{\varphi(y)}\right)$ tends to $f\left(S_{y_{-}}\right)=\{P\}$, which implies that $\varphi(y)$ tends to 0 .

Thus, in case of $d(B) \neq 0$, where $B=(a, b)$, we can define the sign of $B$, which we will denote $\Delta(B)$.

$$
\Delta(B)= \begin{cases}1 & \text { if } \lim _{t \rightarrow a^{+}} \varphi(t)=0 \text { and } \lim _{t \rightarrow b^{-}} \varphi(t)=1 \\ -1 & \text { if } \lim _{t \rightarrow a^{+}} \varphi(t)=1 \text { and } \lim _{t \rightarrow b^{-}} \varphi(t)=0 \\ 0 & \text { otherwise }\end{cases}
$$

Finally, for an arbitrary $B$ we define the degree of $B$ as $\operatorname{deg}(B)=\Delta(B) \cdot d(B)$ (and $\operatorname{deg}(B)=0$ if $d(B)=0$ and $\Delta(B)$ is not defined).

Definition 2.5 A connected component $B$ of $\mathbb{T} \backslash \varphi^{-1}(0)$ will be called a preband ${ }^{1}$ if $\operatorname{deg}(B) \neq 0$. We will denote the set of all prebands of $f$ by $\mathcal{B}(f)$.

Observe that if $B$ is a preband then $\varphi(B)=(0,1)$ and $f\left(\ell^{-1}(B)\right)=\mathbb{S}^{2} \backslash\{P\}$.

Remark 2.6 If $\operatorname{deg}(B)=0$, it may happen that the map $\varphi$ is not continuous at the boundary of $B$. The simplest example is a map $f$ given by

$$
f(x, y, z)=\left(\sqrt{1-(z-1)^{2}}, 0, z\right),
$$

\footnotetext{
1 It would be more natural to use the name band, but in several papers this name is used for the sets like $\ell^{-1}(B)$.
} 
where the sphere is given by the equation $x^{2}+y^{2}+(z-1)^{2}=1$, as in the introduction. Then $\varphi^{-1}(0)=0$, so the only component of $\mathbb{T} \backslash \varphi^{-1}(0)$ is $(0,1)$. However, $\varphi(t)=1 / 2$ for all $t \in(0,1)$, while $\varphi(0)=0$. While in this example the degree of $f$ is 0 , one can easily construct similar examples with arbitrary degree of $f$, based on the same idea.

Lemma 2.7 There are only finitely many prebands.

Proof Suppose that the number of prebands is infinite. Then, there exists a sequence of prebands $\left(B_{i}\right)_{i}$ which converges to some $y_{0} \in A$. We choose a point $Q \in S^{2}$ such that $Q \neq P$. As a consequence of the fact that $\operatorname{deg}\left(B_{i}\right) \neq 0$ we may find a sequence of points $\left(Q_{i}\right)_{i}$ such that

$$
Q_{i} \in \ell^{-1}\left(B_{i}\right) \text { and } f\left(Q_{i}\right)=Q
$$

On the other hand, $\left(Q_{i}\right)_{i}$ (or its subsequence) is convergent and we get: $Q_{i} \underset{i \rightarrow \infty}{\longrightarrow} Q_{0}$ and thus, by (2.4), $f\left(Q_{0}\right)=Q$.

However, $\ell\left(Q_{0}\right)=y_{0}$, which leads to the following contradiction:

$$
0=\varphi\left(y_{0}\right)=\varphi\left(\ell\left(Q_{0}\right)\right)=\ell\left(f\left(Q_{0}\right)\right)=\ell(Q) \neq 0,
$$

where in the third equality we use the formula (2.1).

Note that outside the pole $P$ a paridianal map $f$ can be written in the following form:

$$
f(\alpha, \beta)=\left(\varphi(\alpha), f_{\alpha}(\beta)\right)
$$

where $\alpha$ and $\beta$ are the $y$ - and $x$-titude, respectively, and $f_{\alpha}: \mathbb{S}^{1} \rightarrow \mathbb{S}^{1}$ is defined in (2.2). Here we abuse slightly the notation and treat $\alpha$ and $\beta$ as elements of $\mathbb{T}$ rather than angles from $(0, \pi)$.

Now we establish the connection between the degree of $f$ and the degrees of prebands. We do it first under the additional assumption that $f$ is of class $C^{1}$.

Lemma 2.8 If $f: \mathbb{S}^{2} \rightarrow \mathbb{S}^{2}$ is a $C^{1}$ paridianal map, then its degree is

$$
\operatorname{deg}(f)=\sum_{B \in \mathcal{B}(f)} \operatorname{deg}(B)
$$

Proof As $f$ is a $C^{1}$ map, we may choose $Q \neq P$, a regular value of $f$. Then by the definition

$$
\operatorname{deg}(f)=\sum_{x_{i} \in f^{-1}(Q)} \text { sign det } D f\left(x_{i}\right)
$$

In the neighborhood of any point $x_{i} \in f^{-1}(Q)$ the map $f$ has the form (2.5) for $x_{i}=\left(\alpha_{i}, \beta_{i}\right) \in \mathbb{T} \times \mathbb{T}$. On the other hand, $f$ is a paridianal map and thus $\varphi$ is a function of only one variable $\alpha$. Thus 


$$
D f_{x_{i}}=\left[\begin{array}{cc}
a_{i} & 0 \\
\cdot & b_{i}
\end{array}\right],
$$

where $a_{i} \in \mathbb{R}$ is the derivative of $\varphi$ at $\alpha_{i}$ and $b_{i}=\frac{d f_{\alpha}}{d \beta}\left(\beta_{i}\right)$.

For each fixed $\alpha_{i}$ take the finite set of all $\beta_{i j}$ such $\left(\alpha_{i}, \beta_{i j}\right) \in f^{-1}(Q)$. Then, by the formula (2.7) and taking into account that by Lemma $2.3 d_{S_{\alpha_{i}}}(f)$ is the same for all $\alpha_{i} \in B$ and is equal to $d(B)$, we get

$$
\begin{aligned}
\operatorname{deg}(f) & =\sum_{B} \sum_{x_{i} \in l^{-1}(B)} \operatorname{sign} \operatorname{det} D f\left(x_{i}\right)=\sum_{B}\left(\sum_{\alpha_{i} \in B} \operatorname{sign}\left(a_{i}\right) \cdot \sum_{\beta_{i j} \in S_{\alpha_{i}}} \operatorname{sign}\left(b_{i j}\right)\right) \\
& =\sum_{B}\left(\sum_{\alpha_{i} \in B} \operatorname{sign}\left(a_{i}\right) d(B)\right)=\sum_{B} \Delta(B) \cdot d(B),
\end{aligned}
$$

where the summation is taken over a finite number of components $B$ of the set $\mathbb{T} \backslash \varphi^{-1}(0)$ (for some of them perhaps $\operatorname{deg}(B)=0$ ). This gives us formula (2.6).

Theorem 2.9 If $f: \mathbb{S}^{2} \rightarrow \mathbb{S}^{2}$ is a paridianal map, then formula (2.6) holds.

Proof In order to use Lemma 2.8, we should construct a $C^{1}$ paridianal map, homotopic to $f$, whose prebands have the same degrees as the prebands of $f$.

The first step is to modify $f$ by homotopy to a map $f_{1}$ in such a way that $f_{1}$ differs from $f$ only on the sets $\ell^{-1}(B)$ for components $B$ of $\mathbb{T} \backslash\left\{\varphi^{-1}(0)\right\}$ which are not prebands. The map $f_{1}$ sends each such set $\ell^{-1}(B)$ to $P$. To show that $f_{1}$ is homotopic to $f$, it is enough to look at each $B$ separately. If $\Delta(B)=0$, then it is clear that $f_{1}$ is homotopic to $f$ on the closure of $\ell^{-1}(B)$, because $\varphi$ is homotopic to a constant on the closure of $B$. If $d(B)=0$, then to see the homotopy we note that all maps $f_{y}$, defined by (2.2), can be uniformly homotoped to a constant. When moving from one endpoint of $B$ to the other one, we do not go around the circle, because all maps $f_{y}$ are "anchored" at $P$ (that is, $P$ belongs to all paridians and $f(P)=P$ ).

The second step is to modify $f_{1}$ by homotopy to a map $f_{2}$, by making $f_{2}$ on each circle $\ell^{-1}(\alpha)$ with $\alpha$ in a preband $B$, affinely conjugate to the complex map $z \mapsto z^{d(B)}$ of the unit circle. Of course we keep $f_{2}(P)=P$. This clearly can be done and the prebands of $f_{2}$ are the same as for $f$ and have the same degrees.

The third step is to modify $f_{2}$ by homotopy to a map $f_{3}$, which is smooth at all points which are not mapped to $P$, has the same prebands as $f_{2}$ and the degrees of the prebands are the same. We do it by writing $f_{2}$ in the coordinates $\alpha, \beta$ like in (2.5), that is,

$$
f_{2}(\alpha, \beta)=\left(\varphi_{2}(\alpha), f_{2, \alpha}(\beta)\right)
$$

If we replace in this formula $\varphi_{2}$ by a continuous map $\varphi_{3}$, which differs from $\varphi_{2}$ only at the prebands, and is affine on each preband, we get a map $f_{3}$ with the required properties. 
Observe that $f_{3}$ is already smooth at all points that are not mapped to $P$. To make it smooth and preserve the degree, the number of prebands and the degrees of corresponding prebands, we replace it with a map $f_{4}=g \circ f_{3}$, where $g$ is a smooth paridianal map which is close to identity, maps a small neighborhood of $P$ to $P$, and maps homeomorphically the rest of the sphere onto $\mathbb{S}^{2} \backslash\{P\}$. We get such a map by taking a smooth map $\psi:[0,1] \rightarrow[0,1]$ which maps small neighborhoods of 0 and 1 to 0 and 1 respectively, and is strictly increasing on the rest of the interval. Then we set

$$
g(\alpha, \beta)=(\psi(\alpha), \psi(\beta))
$$

Now Lemma 2.8 applies to $f_{4}$ and we get the formula (2.6) for our original map $f$.

When studying the behavior of $f$ near the pole (and the derivative $D f(P)$ ) we consider the local planar coordinate system near $P$ given by the stereographic projection from the antipole $(0,0,2)$. In this system 0 is a fixed point representing $P$ and circles represent paridians (see Fig. 1). The $x$-axis (with the point at infinity, which we will not mention later) is also a paridian. To simplify the notation we use the same letter $f$ for our map in this coordinate system.

Now we want to compare the number of fixed points of a paridianal map $f$ with its degree if $|\operatorname{deg}(f)|>1$.

We have to show that in each preband the map $\varphi$ has a fixed point (which must be different from 0 and 1 which are in the set $A$ ).

We will say that the pole is a sink if it is asymptotically stable for $f$.

Lemma 2.10 Assume that the pole is a sink and 0 is an endpoint of a preband $B$ with $\Delta(B)=1$. Then there is $\varepsilon>0$ such that if $0<t<\varepsilon$ then $\varphi(t)<t$.

Proof Suppose that arbitrarily close to 0 there is $t$ such that $\varphi(t) \geq t$. Then either $\varphi(t)>t$ for all $t$ sufficiently close to 0 , or arbitrarily close to 0 there are fixed points of $\varphi$.

In the first case, since 0 is an endpoint of a preband, for any $t$ sufficiently close to 0 the map from $\ell^{-1}(t)$ to $\ell^{-1}(\varphi(t))$ is a surjection. Therefore for any $t$ sufficiently close to 0 there is a sequence of positive numbers $t_{n}$, convergent to 0 , such that $f^{n}\left(\ell^{-1}\left(t_{n}\right)\right)=\ell^{-1}(t)$. This means that $P$ is even not Lyapunov stable, a contradiction.

In the second case, if $t$ is a fixed point of $\varphi$, then $P$ is asymptotically stable for $f$ restricted to the circle $\ell^{-1}(t)$, which implies that in $\ell^{-1}(t)$ there is a fixed point of $f$ other than $P$. However, if $t$ is sufficiently small, then trajectories of all points of $\ell^{-1}(t)$ converge to $P$, a contradiction.

Lemma 2.11 Consider a paridianal map $f$ for which the pole is a sink. Assume that $B \neq(0,1)$ is a preband, or $B=(0,1)$ with $|d(B)|>1$. Then there is a fixed point of $\varphi$ in $B$.

Proof We have to show that the graph of $\varphi$ on $B$ crosses the diagonal. This is clear if the closure of $B$ does not contain 0 and 1. Suppose now that one endpoint of $B$ is 0 (with 1 the situation is analogous). 
If $\Delta(B)=-1$ then clearly $\varphi$ has a fixed point in $B$. Suppose that $\Delta(B)=1$. By Lemma 2.10, the point $(t, \varphi(t))$ lies below the diagonal for small $t>0$. Thus, again the graph of $\varphi$ on $B$ crosses the diagonal.

Corollary 2.12 Let $B$ be a preband of a paridianal map f for which the pole is a sink. Then either there is a fixed point of $\varphi$ in $B$, or $|\operatorname{deg}(f)| \leq 1$.

If $f$ is smooth in a neighborhood of $P$ then in important cases $P$ is automatically a sink.

Theorem 2.13 Assume that a paridianal map $f$ is of class $C^{1}$ near the pole and one of the following conditions hold:

(1) there is a preband $B \neq(0,1)$,

(2) $B=(0,1)$ is a preband and $|d(B)|>1$.

Then $\operatorname{Df}(P)=0$, so the pole is a sink.

Proof We use the planar coordinates, so $P$ becomes 0 , and proper paridians become the $x$-axis and circles tangent to it at 0 (see Fig. 1).

We will start by proving that $D f(0)$ maps paridians that are circles to paridians. Let $L_{r}$ be a paridian of diameter $r$. Fix $\varepsilon>0$. By the definition of the derivative, there exists $\delta_{0}$ such that for every vector (point) $v$ if $\|v\| \leq \delta_{0} r$ then

$$
\|f(v)-D f(0)(v)\| \leq \varepsilon\|v\| .
$$

For $\delta \in\left(0, \delta_{0}\right)$, define the map $g_{\delta}$ by $g_{\delta}(v)=f(\delta v) / \delta$. Since $f$ maps paridians to paridians, the set $g_{\delta}\left(L_{r}\right)$ is contained in a paridian. For $v \in L_{r}$ we have $\|\delta v\| \leq \delta r$, and $D f(0)$ is linear, so

$$
\left\|g_{\delta}(v)-D f(0)(v)\right\|=\frac{1}{\delta}\|f(\delta v)-D f(0)(\delta v)\| \leq \frac{1}{\delta} \varepsilon\|\delta v\| \leq \varepsilon r .
$$

Since $\varepsilon>0$ was arbitrary, this means that $D f(0)$ is the uniform limit of maps $g_{\delta}$ as $\delta \rightarrow 0$. Therefore, $D f(0)\left(L_{r}\right)$ is contained in a paridian.

In case (1) there is always a proper paridian that is mapped by $f$ to $P$, but then the derivative $D f(0)$ in $x$-direction is 0 . This direction is an eigendirection of $D f(0)$, so the only possibility for paridians to be mapped by $D f(0)$ to paridians is that $D f(0)=0$.

Let us now consider case (2). Then sufficiently small proper paridians cannot be mapped to the $x$-axis, unless they are mapped to 0 . Therefore, for sufficiently small $\delta$, the set $g_{\delta}\left(L_{r}\right)$ is either a proper paridian other than the $x$-axis, or $\{0\}$. Hence, the same is true for $D f(0)$ instead of $g_{\delta}$. This means that either $\operatorname{det}(D f(0)) \neq 0$ or $D f(0)=0$.

If $\operatorname{det}(D f(0)) \neq 0$, then $f$ is one-to-one in a small neighborhood of 0 . However, since $|d(B)|>1$, in such neighborhood there are paridians, which are mapped by $f$ not in the one-to-one manner. This is a contradiction, so we must have $D f(0)=0$.

Definition 2.14 We will call a paridianal map $f$ sinking if the pole is a sink for $f$.

From Theorems 2.9 and 2.13, we get the following corollary. 
Corollary 2.15 Assume that a paridianal map $f$ is of class $C^{1}$ near the pole and $|\operatorname{deg}(f)|>1$. Then $f$ is sinking.

Remark 2.16 In the class of paridianal maps that are not sinking, we can find simple examples of maps of degree $d$, with $|d|>1$, that have no periodic points except one fixed point and the maps are not differentiable only at that point. Although such examples are already known in literature (cf. [3], Example 2.7), but in our approach they could be produced very easily. Namely, taking the map that is conjugated to $z^{d}$ on each paridian and such that the graph of $\varphi$ is over (or under) the diagonal (for example $\left.\varphi(t)=\frac{1}{2} t(t+1)\right)$, we get a desired map.

A fixed point of $\varphi$ in a preband gives us a proper paridian that is mapped by $f$ to itself. The next step is to estimate the number of fixed points in this paridian, other than $P$, compared to the degree of $f$ restricted to this paridian.

Lemma 2.17 Let $f$ be a paridianal map. Let $B$ be a preband with $d(B) \leq 0$, and let $y \in B$ be a fixed point of $\varphi$. Then there are at least $|d(B)|$ fixed points of $f$, other than $P$, in $\ell^{-1}(y)$.

Proof Any continuous map of a circle to itself of degree $d$ has at least $|d-1|$ fixed points (cf. [13]). Thus, if $d \leq 0$, this number is at least $|d|+1$. Taking into account that in our case one of those points is $P$, we get at least $|d(B)|$ other fixed points in $\ell^{-1}(y)$.

This leaves us with the case when $d(B)>0$, when we have to take into account the local behavior of the map at $P$.

Lemma 2.18 Let $f$ be a sinking paridianal map. Assume that $B \neq(0,1)$ is a preband with $d(B)>0$ or $B=(0,1)$ with $d(B)>1$; and $y \in B$ is a fixed point of $\varphi$. Then there are at least $d(B)$ fixed points of $f$, other than $P$, in $\ell^{-1}(y)$.

Proof As in the Proof of Lemma 2.10, $P$ is asymptotically stable for $f$ restricted to the circle $\ell^{-1}(y)$, and thus, 0 is an attracting fixed point of $f_{y}$ [see (2.2)].

Now consider $\tilde{f}_{y}:[0,1] \rightarrow \mathbb{R}$, the lift of $f_{y}$. The graph of $\tilde{f}_{y}$ intersects $d(B)$ straight lines of the form $y=x+k, k=0, \ldots, d(B)-1$, and thus there must be at least $d(B)$ fixed points of $f_{y}$ other than 0 . This completes the proof.

Now we can get an estimate of the number of fixed points of a sinking paridianal map $f$ with $|\operatorname{deg}(f)|>1$.

Theorem 2.19 Let $f$ be a sinking paridianal map with $|\operatorname{deg}(f)|>1$. Then

$$
\# \operatorname{Fix}(f) \geq|\operatorname{deg}(f)|+1
$$

Proof Since $|\operatorname{deg}(f)|>1$ and by Theorem 2.9, either each preband is different from $(0,1)$, or $B=(0,1)$ is the only preband and $|d(B)|>1$. Thus, by Lemma 2.11 , there is a fixed point of $\varphi$ in every preband. By Lemmas 2.17 and 2.18, we see that there are at least $|d(B)|=|\operatorname{deg}(B)|$ fixed points of $f$, other than $P$, in every preband $B$. Using Theorem 2.9, and remembering that $P$ is also a fixed point, we get (2.9). 
Taking into account Lemma 2.2 in case $|\operatorname{deg}(f)| \leq 1$, we get for an arbitrary degree the following corollary.

Corollary 2.20 If $f$ is a sinking paridianal map, then it has at least $|\operatorname{deg}(f)|$ fixed points.

If $f$ is a sinking paridianal map then $f^{n}$ is also a sinking paridianal map. Moreover, $\operatorname{deg}\left(f^{n}\right)=\operatorname{deg}(f)^{n}$. As a consequence, we obtain the conclusion, in which we obtain (1.1) in a stronger version with the upper limit replaced by the lower limit.

Corollary 2.21 If $f$ is a sinking paridianal map, then $f^{n}$ has a at least $|\operatorname{deg}(f)|^{n}$ fixed points.

Remark 2.22 Let us notice that Theorem 2.19 and Corollaries 2.20 and 2.21 may be presented in more general form, for a larger class of foliations. Namely, let us denote our paridianal foliation by $\mathcal{F}$ and consider a foliation $h(\mathcal{F})$, where $h$ is a homeomorphism of $\mathbb{S}^{2}$. Then, the mentioned theorem and corollaries hold for a map $f$ preserving this foliation, for which $h(P)$ is a sink. This follows from the fact that $h^{-1} \circ f \circ h$ is a sinking paridianal map.

Remark 2.23 In view of Corollary 2.15, in Theorem 2.19 and Corollaries 2.20 and 2.21 we can replace "sinking paridianal maps" by "paridianal maps that are of class $C^{1}$ in a neighborhood of the pole".

\section{Rabbit Maps}

Here we use the rabbit foliation, defined in the introduction, and consider smooth (of class $C^{1}$ ) sphere maps preserving this foliation, the rabbit maps. We denote the family of all rabbit maps by $\mathcal{R}$.

Let us be more precise. The pole is still $P$ and there are two ears, which are the sets given (in the plane, after the stereographic projection, see Fig. 2) in the polar coordinates by $\{(r, \theta): r=c \sin (2 \theta), c \in[0,1]\}$ in the first quadrant and $\{(r, \theta)$ : $r=-c \sin (2 \theta), c \in[0,1]\}$ in the second quadrant. We denote them by $E_{1}$ and $E_{2}$, and their union by $E$. Each ear is foliated by the appropriate curves $r= \pm c \sin (2 \theta)$; $P$ is a singular point and belongs to all leaves. We divide the rest of the sphere, $G=\left(\mathbb{S}^{2} \backslash E\right) \cup\{P\}$ into two areas $G_{1}$ which is the part of $G$ in the first and the second quadrant and $G_{2}$ which is the part in the third and fourth quadrant and define the foliation in the following way. In $G_{1}$ its leaves are given by $r=2 \sin \theta \sqrt{\cos ^{2} \theta+c}$, where $c \geq 0$. In $G_{2}$ the leaves are circles $r=c \sin \theta$. Additionally, the $x$-axis (with the point at infinity) is also a leaf.

Two leaves are very special. They are boundaries of the ears, $\partial E_{1}$ and $\partial E_{2}$. The leaves other than $\partial E_{1}, \partial E_{2}$ and $\{P\}$ will be called regular.

Fix a rabbit map $f$.

Lemma 3.1 If $\operatorname{deg}(f) \neq 0$ then $f(P)=P$.

Proof If $f(P) \neq P$ then, since every leaf contains $P$, the image of the whole sphere is contained in one leaf. Therefore $\operatorname{deg}(f)=0$. 
In the rest of the paper we assume that $\operatorname{deg}(f) \neq 0$. In particular, $f(P)=P$. We will call the leaves of the rabbit foliation simply leaves.

As in the preceding section, we want to define the map $\ell$. However, now we need it only in a one-sided (from the side of $G_{1}$ ) neighborhood $U$ of $\partial E$. There we parametrize the set of leaves by an interval $[0, \alpha)$, for a small $\alpha>0$, where 0 corresponds to the union of two leaves $\partial E_{1}$ and $\partial E_{2}$. Thus, we have a projection $\ell: U \rightarrow[0, \alpha)$. We want to show that there exists $\varepsilon \in(0, \alpha)$ and $\varphi:[0, \varepsilon) \rightarrow[0, \alpha)$ such that $\varphi \circ \ell=\ell \circ f$.

We have the orientation of proper paridians consistent throughout the sphere. Therefore if $L, K$ are leaves other than $\{P\}$, and $f(L) \subset K$, the degree $d_{L}(f)$ of the map $\left.f\right|_{L}: L \rightarrow K$ is well defined.

We start with three lemmas.

Lemma 3.2 Let $\left(L_{n}\right)$ and $\left(K_{n}\right)$ be sequences of leaves other than $\{P\}$, such that $f\left(L_{n}\right) \subset K_{n}, d_{L_{n}}(f) \neq 0$, and the sequence $\left(K_{n}\right)$ converges to $\partial E$ from the side of $G_{1}$. Then the sequence $\left(L_{n}\right)$ also converges to $\partial E$ from the side of $G_{1}$.

Proof Observe that since $d_{L_{n}}(f) \neq 0$, we have $f\left(L_{n}\right)=K_{n}$. If there is a subsequence of $\left(L_{n}\right)$ convergent to a leaf $L$, then $f(L)=\partial E$, but $\partial E$ is not contained in any leaf, a contradiction. However, the only possibility that there is no subsequence of $\left(L_{n}\right)$ convergent to a leaf is that $\left(L_{n}\right)$ converges to $\partial E$ from the side of $G_{1}$.

Lemma 3.3 We have $f(\partial E)=\partial E$. Moreover, either $f\left(\partial E_{1}\right)=\partial E_{1}$ and $f\left(\partial E_{2}\right)=$ $\partial E_{2}$, or $f\left(\partial E_{1}\right)=\partial E_{2}$ and $f\left(\partial E_{2}\right)=\partial E_{1}$. If a leaf $L \subset G_{1}$ is sufficiently close to $\partial E$, then $\left|d_{L}(f)\right|=1$.

Proof Choose a point $x \in \partial E$ and a sequence $\left(x_{n}\right)$ convergent to $x$ from the side of $G_{1}$, which are regular values of $f$. Since $\operatorname{deg}(f) \neq 0$, for each $n$ there is a point $y_{n} \in f^{-1}\left(x_{n}\right)$ such that if $L_{n}$ is the leaf containing $y_{n}$, then $d_{L_{n}}(f) \neq 0$ (if $d_{L_{n}}(f)=0$ then the sum of the signs of the Jacobian of $f$ over all elements of $L_{n} \cap f^{-1}\left(x_{n}\right)$ is zero). The leaf $K_{n}=f\left(L_{n}\right)$ contains $x_{n}$, so the sequence $\left(K_{n}\right)$ also converges to $\partial E$ from the side of $G_{1}$. Then, by Lemma 3.2, the sequence $\left(L_{n}\right)$ converges to $\partial E$ from the side of $G_{1}$. Therefore, $f(\partial E)=\partial E$.

The second statement of the lemma follows from the fact that $f$ maps leaves to leaves. The third statement follows from the second one.

Lemma 3.4 There exists a one-sided (from the side of $G_{1}$ ) neighborhood $U$ of $\partial E$, an interval $[0, \alpha)$, a projection $\ell: U \rightarrow[0, \alpha)$, and a map $\varphi:[0, \varepsilon) \rightarrow[0, \alpha)$ for some $\varepsilon \in(0, \alpha)$, such that:

(a) $\ell^{-1}(0)=\partial E$,

(b) $\ell^{-1}(t)$ is a leaffor $t>0$,

(c) $\varphi \circ \ell=\ell \circ f$,

(d) $\varphi(0)=0$

(e) $\varphi$ is continuous on $[0, \varepsilon)$,

(f) $d_{\ell^{-1}(t)}(f)$ is independent of $t \in(0, \varepsilon)$ and its modulus is 1 .

Proof Existence of $U, \alpha$ and $\ell$ satisfying (a) and (b) is obvious. Since $f$ maps leaves to leaves, it is clear how to define $\varphi$ so that it satisfies (c). By Lemma 3.3, for a sufficiently 
small $\varepsilon>0$, if a leaf $L$ is contained in $\ell^{-1}((0, \varepsilon))$, then $f(L) \subset U$. Therefore $\varphi$ is well defined in some $[0, \varepsilon)$. Property (d) follows from Lemma 3.3. Properties (e) and (f) follow from Lemma 3.3 and continuity of $f$.

Now we can prove the main theorem of this section.

Theorem 3.5 If $f$ is a rabbit map, then $|\operatorname{deg}(f)| \leq 1$. Moreover, it has at least one fixed point.

Proof Assume that $\operatorname{deg}(f) \neq 0$. Choose a point $x \in G_{1}$, which is a regular value of $f$, and lies sufficiently close to $\partial E$ (but far from $P$ ). By Lemma 3.2, all elements of $f^{-1}(x)$ which belong to leaves $L$ such that $d_{L}(f) \neq 0$, lie in $\ell^{-1}((0, \varepsilon))$. By Lemma 3.4 (f) and the same arguments as in the Proof of Lemma 2.8, $\operatorname{deg}(f)$ is equal to the common value of $d_{\ell^{-1}(t)}(f)$ (which has modulus 1 ) multiplied by the sum of the signs of $\varphi^{\prime}$ at the points of $\varphi^{-1}(\ell(x))$. This sum has modulus not larger than 1 , so $|\operatorname{deg}(f)| \leq 1$.

If $\operatorname{deg}(f) \neq 0$, then by Lemma $3.1 P$ is a fixed point of $f$. If $\operatorname{deg}(f)=0$ then considering $L(f)$, the Lefschetz number of $f$, we get $L(f)=1+\operatorname{deg}(f)=1 \neq 0$. Therefore, by Lefschetz fixed point theorem, $f$ also has a fixed point.

Remark 3.6 Let us note that by using arguments similar to those from the Proof of Theorem 2.9, one can prove Theorem 3.5 for continuous maps preserving the rabbit foliation.

From Theorem 3.5 we get an obvious corollary.

Corollary 3.7 If $f$ is a rabbit map and $\operatorname{deg}(f) \neq 0$, then the lower growth rate of the number of fixed points of $f^{n}$ is at least $\log |\operatorname{deg}(f)|$.

Open Access This article is distributed under the terms of the Creative Commons Attribution 4.0 International License (http://creativecommons.org/licenses/by/4.0/), which permits unrestricted use, distribution, and reproduction in any medium, provided you give appropriate credit to the original author(s) and the source, provide a link to the Creative Commons license, and indicate if changes were made.

\section{References}

1. Babenko, I.K., Bogatyi, S.A.: The behavior of the index of periodic points under iterations of a mapping. Math. USSR Izv. 38, 1-26 (1992)

2. Boroński, J.P.: A fixed point theorem for the pseudo-circle. Topol. Appl. 158, 775-778 (2011)

3. Blokh, A., Oversteegen, L.: A fixed point theorem for branched covering maps of the plane. Fund. Math. 206, 77-111 (2009)

4. Brumfiel, G.: 'Overwhelming' evidence for monopoles. Nature 3 (2009). https://doi.org/10.1038/news. 2009.881

5. Graff, G., Jezierski, J.: On the growth of the number of periodic points for smooth self-maps of a compact manifold. Proc. Am. Math. Soc. 135(10), 3249-3254 (2007)

6. Graff, G., Jezierski, J.: Minimal number of periodic points for smooth self-maps of $S^{3}$. Fund. Math. 204, 127-144 (2009)

7. Graff, G., Misiurewicz, M., Nowak-Przygodzki, P.: Periodic points of latitudinal maps of the $m$ dimensional sphere. Discrete Cont. Dyn. Syst. A 36, 6187-6199 (2016) 
8. Graff, G., Misiurewicz, M., Nowak-Przygodzki, P.: Shub's conjecture for smooth longitudinal maps of $S^{m}$. J. Differ. Equ. Appl. 24, 1044-1054 (2018)

9. Hernández-Corbato, L., Ruiz del Portal, F.R.: Fixed point indices of planar continuous maps. Discrete Contin. Dyn. Syst. 35, 2979-2995 (2015)

10. Honorato, G., Iglesias, J., Portela, A., Rovella, A., Valenzuela, F., Xavier, J.: On the growth rate inequality for periodic points in the two sphere (2017). preprint arXiv:1707.06144v1

11. Iglesias, J., Portela, A., Rovella, A., Xavier, J.: Dynamics of annulus maps II: periodic points for coverings. Fund. Math. 235(3), 257-276 (2016)

12. Iglesias, J., Portela, A., Rovella, A., Xavier, J.: Dynamics of annulus maps III: completeness. Nonlinearity 29, 2641-2656 (2016)

13. Jiang, B.J.: Lectures on the Nielsen Fixed Point Theory, Contemp. Math. 14. Amer. Math. Soc., Providence (1983)

14. Lloyd, N.G.: Degree Theory, Cambridge Tracts in Mathematics, no. 73. Cambridge University Press, Cambridge (1978)

15. Misiurewicz, M.: Periodic points of latitudinal maps. J. Fixed Point Theory Appl. 16(1-2), 149-158 (2014)

16. Pugh, C., Shub, M.: Periodic points on the 2-sphere. Discrete Contin. Dyn. Syst. 34, 1171-1182 (2014)

17. Shub, M.: Dynamical systems, filtration and entropy. Bull. Am. Math. Soc. 80, 27-41 (1974)

18. Shub, M.: All, most, some differentiable dynamical systems. In: Proceedings of the International Congress of Mathematicians, Madrid, Spain, pp. 99-120. European Math. Society (2006)

19. Shub, M., Sullivan, P.: A remark on the Lefschetz fixed point formula for differentiable maps. Topology 13, 189-191 (1974)

Publisher's Note Springer Nature remains neutral with regard to jurisdictional claims in published maps and institutional affiliations. 